\title{
Efficacy and safety of febuxostat in elderly female patients
}

This article was published in the following Dove Press journal:

Clinical Interventions in Aging

4 September 2014

Number of times this article has been viewed

\author{
Tomohiro Mizuno ${ }^{1,2}$ \\ Takahiro Hayashi ${ }^{3}$ \\ Sayo Hikosaka' \\ Yuka Shimabukuro' \\ Maho Murase' \\ Kazuo Takahashi ${ }^{2}$ \\ Hiroki Hayashi \\ Yukio Yuzawa ${ }^{2}$ \\ Tadashi Nagamatsu' \\ Shigeki Yamada ${ }^{3}$ \\ 'Department of Analytical \\ Pharmacology, Graduate School \\ of Pharmacy, Meijo University, \\ Nagoya, Japan; ${ }^{2}$ Department of \\ Nephrology, School of Medicine, \\ Fujita Health University, Toyoake, \\ Japan; ${ }^{3}$ Department of Clinical \\ Pharmacy, School of Medicine, Fujita \\ Health University, Toyoake, Japan
}

Correspondence: Takahiro Hayashi

Department of Clinical Pharmacy, School

of Medicine, Fujita Health University,

I-98, Dengakugakubo, Kutsukake,

Toyoake, Aichi 470-I 192, Japan

Tel +8I 562932157

Fax +8I 562934537

Email taka-h@fujita-hu.ac.jp
Background: Maintenance of low serum urate levels is important for the management of gout. Achieving the recommended serum urate levels of less than $6.0 \mathrm{mg} / \mathrm{dL}$ is difficult in elderly (65 years of age or older) patients with renal impairment. Xanthine oxidase inhibitors allopurinol and febuxostat are used for this purpose. Although febuxostat had been shown to be efficacious in elderly patients, its safety and efficacy in elderly female patients with hyperuricemia remain unclear.

Objective: The aim of this study was to assess the efficacy and safety of febuxostat in elderly female patients.

Methods: We studied a retrospective cohort study. The study included elderly Japanese patients (65 years of age or older) who were treated with febuxostat at Fujita Health University Hospital from January 2012 to December 2013. The treatment goal was defined as achievement of serum urate levels of $6.0 \mathrm{mg} / \mathrm{dL}$ or lower within 16 weeks; this was the primary endpoint in the present study. Adverse events of febuxostat were defined as more than twofold increases in Common Terminology Criteria for adverse events scores from baseline.

Results: We evaluated 82 patients treated with febuxostat during the observation period and classified them into male $(n=53)$ and female $(n=29)$ groups. The mean time to achievement of the treatment goal was significantly shorter in the female group (53 days) than in the male group (71 days). There were no significant differences in adverse events between the 2 groups.

Conclusion: Our findings suggest that the efficacy of febuxostat in elderly female patients is superior to that in elderly male patients and that the safety is equivalent.

Keywords: febuxostat, elderly female patients, hyperuricemia

\section{Introduction}

Maintenance of low serum urate (sUA) levels is important for the management of gout. ${ }^{1}$ Achieving the recommended sUA levels of less than $6.0 \mathrm{mg} / \mathrm{dL}^{2,3}$ is difficult in elderly ( 65 years of age and older) patients with renal impairment. The xanthine oxidase inhibitors allopurinol and febuxostat have been widely used for this purpose.

Allopurinol has been used as a first-line drug for the treatment of hyperuricemia. ${ }^{3}$ Adverse reactions such as hepatic disorder, hypersensitivity vasculitis, and bone marrow depression have been reported..$^{4-8}$ Moreover, the dose of allopurinol requires reduction according to the degree of renal impairment. ${ }^{9,10}$

Febuxostat is a nonpurine selective inhibitor of xanthine oxidase that forms a very stable interaction with both the oxidized and reduced forms of the enzyme. ${ }^{11}$ Becker et al reported superior efficacy of febuxostat compared with that of allopurinol in diabetic gout patients. ${ }^{12}$ However, the efficacy of febuxostat was not superior to that of allopurinol in the Febuxostat versus Allopurinol Controlled Trial, ${ }^{6}$ and there was no significant difference between the two drugs with respect to the rate of adverse events (AEs). ${ }^{6,13,14}$ 
Febuxostat has been shown to be efficacious in elderly patients; $;^{13}$ however, the efficacy and safety of febuxostat in elderly female patients with hyperuricemia remain unclear. To promote proper use of febuxostat, we investigated the efficacy and safety of febuxostat in elderly female patients.

\section{Methods}

\section{Subjects}

Elderly Japanese patients ( 65 years of age or older) who were treated with febuxostat at Fujita Health University Hospital from January 2012 to December 2013 were included in the study. Patients treated with hemodialysis or peritoneal dialysis, those treated with anticancer or immunosuppressive therapies, those with baseline sUA levels of less than $7.0 \mathrm{mg} / \mathrm{dL}$, and those for whom sUA levels were not measured within 16 weeks after starting febuxostat therapy were excluded. The patients were divided into two groups: males and females.

\section{Investigations}

This retrospective cohort study used information from the electronic medical records of Fujita Health University Hospital. Renal function in patients was evaluated using estimated glomerular filtration rate values. These values were calculated using the modified isotope dilution mass spectrometry-traceable Modification of Diet in Renal Disease Study equation. ${ }^{15}$ The treatment goal was defined as achievement of sUA levels of $6.0 \mathrm{mg} / \mathrm{dL}$ or less ${ }^{16}$ within 16 weeks; this was the primary end point in the present study. AEs of febuxostat were defined as more than twofold increases in Common Terminology Criteria for Adverse Events scores compared with baseline. We focused on AEs commonly observed at high frequency when using xanthine oxidase inhibitors. ${ }^{6-8,17}$ Among them, leukopenia hepatic disorder, thrombocytopenia, and anemia were selected for use as secondary end points in the present study. This study was approved by the Ethics Board of Fujita Health University Hospital.

\section{Statistical analysis}

Continuous data are presented as the mean (range), and nominal data are presented as percentages. Continuous and nominal data were analyzed with Student's $t$-test and chi-square test, respectively. The mean time to achievement of the treatment goal was measured from the initiation of febuxostat treatment until the first observation of sUA levels of $6.0 \mathrm{mg} / \mathrm{dL}$ or less. Time-to-event curves were plotted using the Kaplan-Meier method, and comparisons among groups were performed with the log-rank test. In these tests, a twosided $P$-value of less than 0.05 was considered significant. SPSS version 22.0 software (IBM Corporation, Armonk, NY, USA) was used for statistical analysis.

\section{Results}

We evaluated 82 patients treated with febuxostat during the observation period and classified them into male $(n=53)$ and female $(n=29)$ groups. Their baseline characteristics and comorbidities are listed in Table 1 . The mean body weight and body surface area of the female group were significantly lower than those of the male group. Baseline renal function was lower in the female group, whereas liver function and sUA levels were similar between the 2 groups. Morbidity rates for hypertension and diabetes mellitus were not significantly different between the 2 groups. The incidence of a history of cardiovascular events was also similar in the groups.

The results of mean time to achievement of the treatment goal are presented in Figure 1. The mean time in the female group (53 days; 95\% confidence interval, 39-68 days) was significantly shorter than that in the male group (71 days; 95\% confidence interval: $60-83$ days).

AEs after treatment with febuxostat are indicated in Table 2, and the grades of AEs are shown in Table 3. There were no significant differences in all terms between the 2 groups.

\section{Discussion}

The dose of febuxostat commonly prescribed in Japan $(10-20 \mathrm{mg} /$ day) is lower than that prescribed in other countries, and there was a time lag preceding the drug's introduction in Japan. Moreover, the majority of patients with hyperuricemia are male. Thus, clinical evidence of the suitability of febuxostat for elderly female patients in Japan is less than that accumulated in many other countries. To increase the usefulness of febuxostat, we evaluated the efficacy and safety of febuxostat in elderly female patients in Japan. Although achievement of the treatment goal was difficult in patients with renal impairment, more than $70 \%$ of the female patients achieved sUA levels of $6.0 \mathrm{mg} / \mathrm{dL}$ or less within 16 weeks. Furthermore, the mean time to achievement of the treatment goal was shorter in female patients than in male patients. These results suggest that the efficacy of febuxostat is superior in elderly female patients.

Khosravan et al concluded that neither age nor sex has a clinically significant effect on the pharmacokinetics, 
Table I Baseline characteristics and comorbidities

\begin{tabular}{|c|c|c|c|}
\hline Characteristics and comorbidities & Male $(n=53)$ & Female $(n=29)$ & $P$-value \\
\hline Age (range), years & $75(65-89)$ & $78(65-92)$ & 0.160 \\
\hline Body weight (range), kg & $58(38-79)$ & $46(32-72)$ & $<0.001$ \\
\hline Body surface area (range), $\mathrm{m}^{2}$ & $1.6(1.2-2.0)$ & $1.4(1.1-1.8)$ & $<0.001$ \\
\hline Febuxostat dose (range), $\mathrm{mg} /$ day & $13(10-20)$ & $13(10-20)$ & 0.827 \\
\hline 10 mg/day (\%) & $36(68)$ & $19(66)$ & \\
\hline 20 mg/day (\%) & $17(32)$ & $10(35)$ & 0.824 \\
\hline Serum creatinine (range) & $2.7(0.8-7.5)$ & $2.5(0.8-7.0)$ & 0.531 \\
\hline eGFR (range), $\mathrm{mL} / \mathrm{min}$ & $25(6.0-69)$ & $18(4.0-35)$ & 0.014 \\
\hline ALT (range), IU/L & $18(3.0-75)$ & $16(4.0-100)$ & 0.629 \\
\hline AST (range), IU/L & $23(8.0-73)$ & $26(12-100)$ & 0.468 \\
\hline BUN (range), mg/dL & $40.1(\mathrm{II} . \mathrm{I}-84.0)$ & $40.9(10.8-101.7)$ & 0.842 \\
\hline Serum urate (range), $\mathrm{mg} / \mathrm{dL}$ & $9.4(7.3-15.3)$ & $9.5(7.1-14.7)$ & 0.783 \\
\hline$<9.0(\%)$ & $22(42)$ & $10(34)$ & 0.588 \\
\hline 9.0 to $<10.0(\%)$ & $18(34)$ & $9(31)$ & \\
\hline 10.0 to $<\mathrm{II} .0(\%)$ & $8(15)$ & $8(28)$ & \\
\hline$\geq \mid \mathrm{I} .0(\%)$ & $5(9)$ & $2(7)$ & \\
\hline Hematocrit (range), \% & $32.4(19.6-46.7)$ & $31.9(22.5-41.0)$ & 0.677 \\
\hline Hemoglobin (range), g/dL & $10.8(6.8-15.5)$ & $10.4(7.5-13.5)$ & 0.450 \\
\hline Erythrocyte (range), $\times 10^{3}$ & $3.5(2.1-5.4)$ & $3.4(2.59-4.36)$ & 0.583 \\
\hline Leukocyte (range), $\times 10^{3}$ & $6.2(1.0-14.7)$ & $6.1(3.1-10.8)$ & 0.806 \\
\hline Platelet (range), $\times 10^{4} / \mu \mathrm{L}$ & I8.4 (2.8-39.1) & I8.I (I.86-55.0) & 0.886 \\
\hline Hypertension (\%) & $47(88.7)$ & $25(86.2)$ & 0.979 \\
\hline Diabetes mellitus (\%) & $31(58.5)$ & $18(62.1)$ & 0.752 \\
\hline Cardiovascular events (\%) & $40(75.5)$ & $22(75.9)$ & 0.968 \\
\hline
\end{tabular}

Note: To establish $P$-values, the $t$-test or $\chi^{2}$ test were used.

Abbreviations: eGFR, estimated glomerular filtration rate; ALT, alanine aminotransferase; AST, aspartate aminotransferase; BUN, blood urea nitrogen.

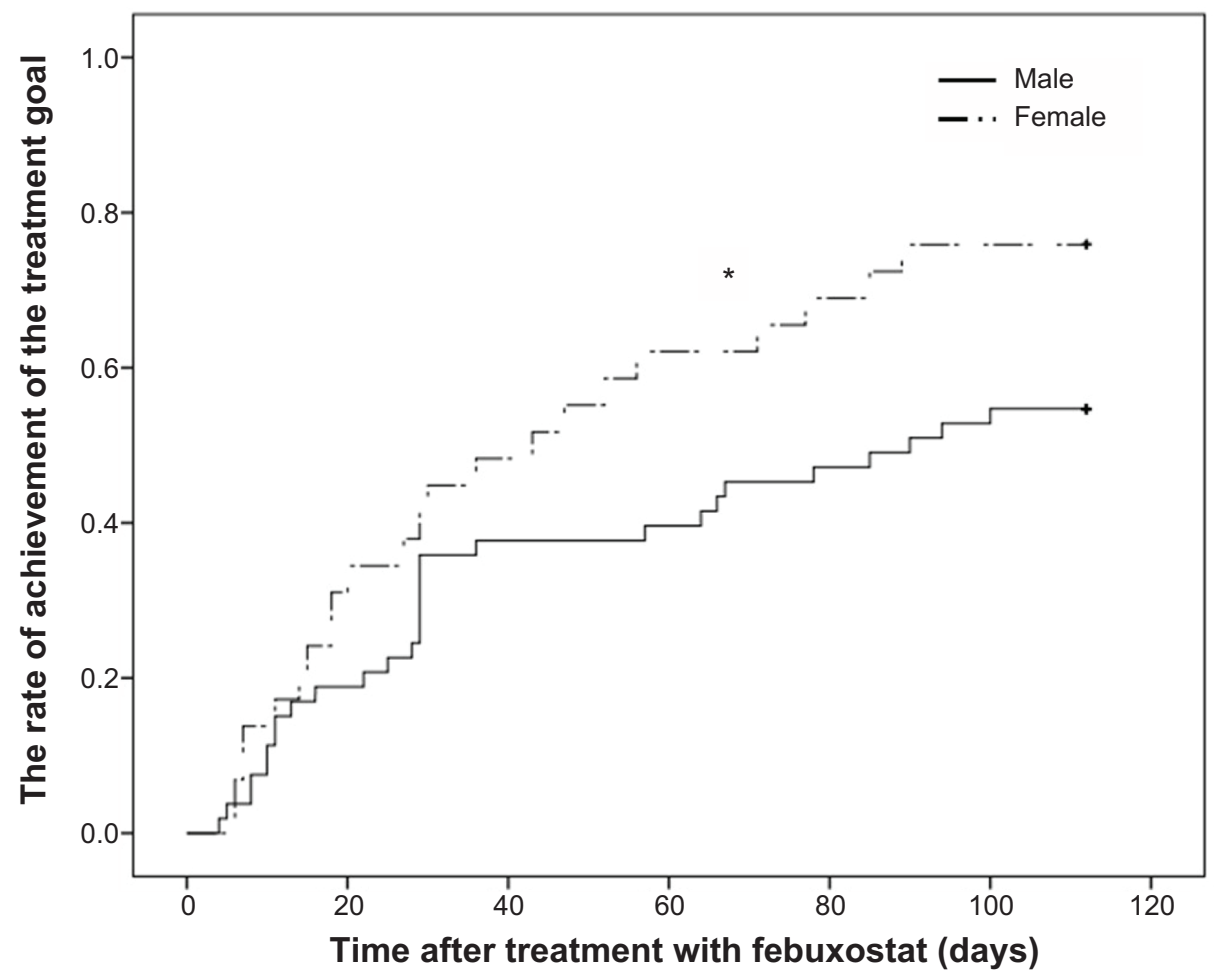

Figure I Mean time to achievement of the treatment goal.

Notes: The mean times to achieve serum urate levels of $6.0 \mathrm{mg} / \mathrm{dL}$ or less in the male and female groups were 71 days ( $95 \%$ confidence interval, $60-83$ days) and 53 days ( $95 \%$ confidence interval, 39-68 days), respectively. $* P=0.047$, male versus female, log-rank test. 
Table 2 Adverse events after treatment of elderly male and female patients with febuxostat

\begin{tabular}{llll}
\hline Characteristic & $\begin{array}{l}\text { Male } \\
(\mathbf{n = 5 3 )}\end{array}$ & $\begin{array}{l}\text { Female } \\
(\mathbf{n = 2 9 )}\end{array}$ & P-value \\
\hline $\begin{array}{l}\text { Number of patients } \\
\text { with adverse events (\%) }\end{array}$ & $5(9.4)$ & $5(17.2)$ & 0.496 \\
Leucopenia (\%) & $2(3.8)$ & I $(3.4)$ & 0.589 \\
Hepatic disorder (\%) & $2(3.8)$ & $2(6.9)$ & 0.530 \\
Thrombocytopenia (\%) & I (I.9) & I (3.4) & 0.756 \\
Anemia (\%) & $3(5.7)$ & I (3.4) & 0.927 \\
\hline
\end{tabular}

pharmacodynamics, or safety of febuxostat. ${ }^{18}$ Their results are in conflict with those of the present study. Khosravan et al investigated these parameters in healthy subjects and assessed the parameters after 7 days, ${ }^{18}$ whereas the present study focused on elderly patients using low doses of febuxostat and observed the parameters for 16 weeks. Therefore, their subjects, doses, and duration of evaluation were different from those of the present study. Moreover, renal function and body surface area levels in female patients in the present study were low; thus, the blood concentrations of febuxostat are expected to have been higher than those observed in the previous study. Some previous reports have suggested that the efficacy of febuxostat ${ }^{13,19,20}$ increases in a dose-dependent manner, whereas its safety in elderly patients is not affected by high doses. ${ }^{13}$ Although the blood concentration of febuxostat may be increased in female patients, the incidence of AEs in female patients was similar to that in male patients. Thus, our results are consistent with those of previous reports. These results suggest that a low dose of febuxostat is safe and efficacious in elderly female patients.

Our study has some limitations. This study employed a retrospective design, and the number of subjects was small. Therefore, larger, prospective, multicenter studies (eg, Febuxostat versus Allopurinol Controlled Trial) will be needed to further validate our results. In addition, the blood concentration of febuxostat was not measured. Further studies that focus on the pharmacodynamics of febuxostat in elderly patients will be needed.

Table 3 Grades of adverse events after treatment with febuxostat

\begin{tabular}{llll}
\hline $\begin{array}{l}\text { Grade } \\
\text { classification }\end{array}$ & $\begin{array}{l}\text { Male } \\
(\mathbf{n}=\mathbf{5 3})\end{array}$ & $\begin{array}{l}\text { Female } \\
(\mathbf{n}=\mathbf{2 9})\end{array}$ & P-value \\
\hline Grade 2 (\%) & $2(3.8)$ & $3(10.3)$ & 0.480 \\
Grade 3 (\%) & $4(7.6)$ & $2(6.9)$ & 0.737 \\
Grade 4 (\%) & $0(0)$ & $0(0)$ & - \\
\hline
\end{tabular}

\section{Conclusion}

In the present study, we demonstrated that the efficacy of febuxostat in elderly female patients is superior to that in elderly male patients, whereas the safety is equivalent.

\section{Disclosure}

The authors report no conflicts of interest in this work.

\section{References}

1. Bardin T, Richette P. New ACR guidelines for gout management hold some surprises. Nat Rev Rheumatol. 2013;9(1):9-11.

2. Zhang W, Doherty M, Bardin T, et al; EULAR Standing Committee for International Clinical Studies Including Therapeutics. EULAR evidence based recommendations for gout. Part II: management. Report of a task force of the EULAR Standing Committee for International Clinical Studies Including Therapeutics (ESCISIT). Ann Rheum Dis. 2006;65(10):1312-1324.

3. Khanna D, Fitzgerald JD, Khanna PP, et al; American College of Rheumatology. 2012 American College of Rheumatology guidelines for management of gout. Part 1: systematic nonpharmacologic and pharmacologic therapeutic approaches to hyperuricemia. Arthritis Care Res (Hoboken). 2012;64(10):1431-1446.

4. Fagugli RM, Gentile G, Ferrara G, Brugnano R. Acute renal and hepatic failure associated with allopurinol treatment. Clin Nephrol. 2008; 70(6):523-526

5. Halevy S, Ghislain PD, Mockenhaupt M, et al; EuroSCAR Study Group. Allopurinol is the most common cause of Stevens-Johnson syndrome and toxic epidermal necrolysis in Europe and Israel. $J$ Am Acad Dermatol. 2008;58(1):25-32.

6. Becker MA, Schumacher HR Jr, Wortmann RL, et al. Febuxostat compared with allopurinol in patients with hyperuricemia and gout. N Engl J Med. 2005;353(23):2450-2461.

7. Stolbach L, Begg C, Bennett JM, et al. Evaluation of bone marrow toxic reaction in patients treated with allopurinol. JAMA. 1982; 247(3):334-336.

8. Paisansinsup T, Breitenstein MK, Schousboe JT. Association between adverse reactions to allopurinol and exposures to high maintenance doses: implications for management of patients using allopurinol. J Clin Rheumatol. 2013;19(4):180-186.

9. Hande KR, Noone RM, Stone WJ. Severe allopurinol toxicity. Description and guidelines for prevention in patients with renal insufficiency. Am J Med. 1984;76(1):47-56.

10. Perez-Ruiz F, Hernando I, Villar I, Nolla JM. Correction of allopurinol dosing should be based on clearance of creatinine, but not plasma creatinine levels: another insight to allopurinol-related toxicity. J Clin Rheumatol. 2005;11(3):129-133.

11. Okamoto K, Eger BT, Nishino T, Kondo S, Pai EF, Nishino T. An extremely potent inhibitor of xanthine oxidoreductase. Crystal structure of the enzyme-inhibitor complex and mechanism of inhibition. $J$ Biol Chem. 2003;278(3):1848-1855.

12. Becker MA, MacDonald PA, Hunt BJ, Jackson RL. Diabetes and gout: efficacy and safety of febuxostat and allopurinol. Diabetes Obes Metab. 2013;15(11):1049-1055.

13. Jackson RL, Hunt B, MacDonald PA. The efficacy and safety of febuxostat for urate lowering in gout patients $\geq 65$ years of age. $B M C$ Geriatr. 2012;12(1):11.

14. Becker MA, Schumacher HR, Espinoza LR, et al. The urate-lowering efficacy and safety of febuxostat in the treatment of the hyperuricemia of gout: the CONFIRMS trial. Arthritis Res Ther. 2010; 12(2):R63. 
15. Matsuo S, Imai E, Horio M, et al; Collaborators developing the Japanese equation for estimated GFR. Revised equations for estimated GFR from serum creatinine in Japan. Am J Kidney Dis. 2009;53(6): 982-992.

16. Iseki K, Ikemiya Y, Inoue T, Iseki C, Kinjo K, Takishita S. Significance of hyperuricemia as a risk factor for developing ESRD in a screened cohort. Am J Kidney Dis. 2004;44(4):642-650.

17. Kobayashi S, Ogura M, Hosoya T. Acute neutropenia associated with initiation of febuxostat therapy for hyperuricaemia in patients with chronic kidney disease. J Clin Pharm Ther. 2013;38(3):258-261.

18. Khosravan R, Kukulka MJ, Wu JT, Joseph-Ridge N, Vernillet L. The effect of age and gender on pharmacokinetics, pharmacodynamics, and safety of febuxostat, a novel nonpurine selective inhibitor of xanthine oxidase. J Clin Pharmacol. 2008;48(9):1014-1024.
19. Zhang M, Di X, Xu L, et al. Pharmacokinetics and pharmacodynamics of febuxostat under fasting conditions in healthy individuals. Exp Ther Med. 2014;7(2):393-396.

20. Khosravan R, Grabowski BA, Wu JT, Joseph-Ridge N, Vernillet L. Pharmacokinetics, pharmacodynamics and safety of febuxostat, a nonpurine selective inhibitor of xanthine oxidase, in a dose escalation study in healthy subjects. Clin Pharmacokinet. 2006;45(8):821-841.

\section{Clinical Interventions in Aging}

\section{Publish your work in this journal}

Clinical Interventions in Aging is an international, peer-reviewed journal focusing on evidence-based reports on the value or lack thereof of treatments intended to prevent or delay the onset of maladaptive correlates of aging in human beings. This journal is indexed on PubMed Central, MedLine,

\section{Dovepress}

CAS, Scopus and the Elsevier Bibliographic databases. The manuscript management system is completely online and includes a very quick and fair peer-review system, which is all easy to use. Visit http://www.dovepress. $\mathrm{com} /$ testimonials.php to read real quotes from published authors. 